\title{
First E- and D-region incoherent scatter spectra observed over Jicamarca
}

\author{
J. L. Chau ${ }^{1}$ and E. Kudeki ${ }^{2}$ \\ ${ }^{1}$ Radio Observatorio de Jicamarca, Instituto Geofísico del Perú, Lima \\ ${ }^{2}$ Department of Electrical and Computer Engineering, University of Illinois, Urbana-Champaign, Illinois, USA \\ Received: 17 August 2005 - Revised: 8 March 2006 - Accepted: 27 March 2006 - Published: 3 July 2006 \\ Part of Special Issue “The 11th International Symposium on Equatorial Aeronomy (ISEA-11), Taipei, May 2005”
}

\begin{abstract}
We present here the first Jicamarca observations of incoherent scatter radar (ISR) spectra detected from E- and D-region altitudes. In the past such observations have not been possible at Jicamarca due a combined effect of strong equatorial electrojet (EEJ) clutter and hardware limitations in the receiving system. The observations presented here were made during weak EEJ conditions (i.e., almost zero zonal electric field) using an improved digital receiving system with a wide dynamic range and a high data throughput.

The observed ISR spectra from E- and D-region altitudes are, as expected, narrow and get even narrower with decreasing altitude due to increasing ion-neutral collision frequencies. Therefore, it was possible to obtain accurate spectral measurements using a pulse-to-pulse data analysis. At lower altitudes in the D-region where signal correlation times are relatively long we used coherent integration to improve the signal-to-noise ratio of the collected data samples. The spectral estimates were fitted using a standard incoherent scatter (IS) spectral model between 87 and $120 \mathrm{~km}$, and a Lorentzian function below $110 \mathrm{~km}$. Our preliminary estimates of temperature and ion-neutral collisions frequencies above $87 \mathrm{~km}$ are in good agreement with the MSISE-90 model. Below $87 \mathrm{~km}$, the measured spectral widths are larger than expected, causing an overestimation of the temperatures, most likely due to spectral distortions caused by atmospheric turbulence.
\end{abstract}

Keywords. Ionosphere (Equatorial ionosphere; Plasma temperature and density; Ionospheric irregularities)

\section{Introduction}

The first incoherent scatter radar (ISR) measurements at the Jicamarca Radio Observatory (JRO) were conducted in 1961 (Bowles et al., 1962). Theoretical and experimental advances in incoherent scatter radar technique since then were accompanied at JRO, located near Lima, Peru, with increasingly

Correspondence to: J. L. Chau

(jchau@jro.igp.gob.pe) more sophisticated measurements of the F-region electron densities, ion and electron temperatures, ion composition, as well as zonal and vertical electric fields (e.g., Farley, 1991; Kudeki et al., 1999; Hysell, 2000, and references therein). Yet, it has not been possible, until the experiment to be described here, to exploit the incoherent scatter technique at JRO to study the equatorial lower ionosphere below $200 \mathrm{~km}$ altitude. The main reason for this has been the following coherent echo sources above Jicamarca which interfere with lower-ionospheric incoherent scatter:

- Equatorial electrojet (EEJ): VHF radar echoes from the electrojet are 2 to 6 orders of magnitude stronger than ISR echoes, confined to the 95-110 (90-140) km region during the day (night), and come from field-aligned irregularities. During the day, these echoes get weaker or disappear when the zonal electric field is close to zero or reverses direction (e.g., Farley, 1985).

- 150-km irregularities: Echoes from the 150-km region are weaker than EEJ echoes but still 1 to 3 orders of magnitude stronger than ISR returns. They occur only during the day and come mainly from field aligned irregularities organized in narrow layers between 140$170 \mathrm{~km}$ altitudes (e.g., Kudeki and Fawcett, 1993). Recently, Chau (2004) have reported the observations of these echoes also at angles slightly off perpendicular to B. Occasionally, relatively strong echoes occur at these altitudes at night, but they are associated to equatorial spread $F$ events (ESF) (e.g., Woodman and Chau, 2001).

- Mesospheric turbulence: Mesospheric echoing layers at $50 \mathrm{MHz}$ caused by neutral turbulence are formed during the day between 55 and $85 \mathrm{~km}$. The echoes are 1-3 orders of magnitude stronger than ISR echoes at similar altitudes, and are organized in very narrow layers (e.g., Woodman and Guillén, 1974; Kudeki, 1988) with widths ranging from less than $100 \mathrm{~m}$ up to few $\mathrm{kms}$ (Sheth et al., 2006).

Published by Copernicus GmbH on behalf of the European Geosciences Union. 
Table 1. Radar Parameters for Faraday experiments on 13 December 2004 .

\begin{tabular}{lcc}
\hline Parameter & Value & Units \\
\hline Inter pulse period (IPP) & 420 & $\mathrm{~km}$ \\
Pulse width & 21 & $\mathrm{~km}$ \\
Binary code & 28 & $\mathrm{bits}$ \\
Receiver Bandwidth & 5 & $\mu \mathrm{s}$ \\
Sampling rate & $5(0.75)$ & $\mu \mathrm{s}(\mathrm{km})$ \\
Initial range & 50 & $\mathrm{~km}$ \\
Number of samples & 456 & \\
Number of complex channels & 2 & \\
Transmitter peak power & 2 & $\mathrm{MW}$ \\
\hline
\end{tabular}

- Meteors: Echoes from meteors could come from meteor heads as well as non-specular and specular meteor trails. At Jicamarca, the first two type of echoes occur between 90 and $110 \mathrm{~km}$, and head echoes occur mainly around sunrise (e.g., Chau and Woodman, 2004). Non-specular trails can last as long as 5 min (e.g., Chapin and Kudeki, 1994) and they come from irregularities perpendicular to B. Specular meteor trails are seldom observed with the Jicamarca ISR due to the narrow beams employed.

Late in the afternoon of 13 December 2004, the Jicamarca radar was being operated with the recently completed wide dynamic-range receivers in a relatively high-resolution and high-sensitivity mode when the zonal electric field that drives the equatorial electrojet vanished for a short period. The radar return samples collected during that period from the D- and E-region altitudes exhibit the first uncluttered and clearcut signatures of lower-ionospheric incoherent scatter detected at JRO. A pulse-to-pulse analysis of the scattered signal samples revealed unaliased Doppler spectra below an altitude of about $110 \mathrm{~km}$, consistent with theoretical expectations for the collision dominated E- and D-regions. The radar spectra to be presented in this paper are not only the first recorded ISR spectra at JRO from D- and E-regions, but also the first for the same heights at any equatorial latitude inside the EEJ belt, i.e., $\pm 3^{\circ}$ with respect to the magnetic equator. Moreover, due to its carrier frequency of $50 \mathrm{MHz}-$ the lowest of all the ISRs - Jicamarca is the only ISR that can measure the E-region with pulse-to-pulse techniques, allowing cleaner spectral estimations than possible anywhere else.

The paper is organized as follows: Sect. 2 describes the experimental setup while in Sect. 3 we present a brief summary of incoherent scatter theory relevant for lower ionospheric heights. D- and E-region spectral measurements are presented in Sect. 4 along with the results of fitting the observed spectra to ISR spectrum models. In Sect. 5 we discuss the observational results and our model fits and, finally, in Sect. 6 we present our conclusions.

\section{Experimental setup}

D- and E-region backscatter measurements described above were performed using the standard Faraday rotation configuration at JRO (e.g., Farley, 1969) except for the pulse waveform details. The radar beam was pointed along the antennaplane on-axis direction, which is nearly vertical and makes an angle of $88.11^{\circ}$ with respect to the nearly horizontal geomagnetic field $\mathbf{B}$ pointing to the north. In this setup the strongest two-way sidelobe of the radar beam that points perpendicular to $\mathbf{B}$ is $\sim 30 \mathrm{~dB}$ weaker than the main beam. Contrary to previous Faraday experiments, a shorter inter pulse period (IPP) $(2.8 \mathrm{~ms}$ instead of $10 \mathrm{~ms})$ and a finer range resolution $(0.75 \mathrm{~km}$ instead of $15 \mathrm{~km})$ were used during the observations. The main parameters of the experiment are presented in Table 1 . We made a full use of the duty cycle available at JRO ( $\sim 5 \%)$ by employing a 28 -bit binary code (Gray and Farley, 1973) with a $0.75 \mathrm{~km}$ baud width.

Two circular polarizations, right- and left-handed, were transmitted and received during the experiment. The results presented here corresponds to one of the circular polarized channels. Since the observed spectra have the same shape in both polarizations at E-region heights and below, statistical fluctuations in the measured spectra can be further reduced by averaging the estimates from the two polarizations (not done here, however). Total power measurements and absolute electron density estimates obtained using both polarizations are reported in Chau and Woodman (2005).

\section{Background parameters and spectral models}

In this section we summarize the main background parameters for the E- and D-region ionosphere needed to understand the incoherent scattered radar spectra presented in the following section. We also specify the spectral model equations used to fit the measured spectra. Since our measurements are confined to daytime, we only focus on daytime conditions.

The shape of incoherent scattered signal spectrum from the low-altitude ionosphere below $110 \mathrm{~km}$ is mainly controlled by ion-neutral collisions. The dominant ions to be considered are $\mathrm{NO}^{+}(30 \mathrm{amu})$ and $\mathrm{O}_{2}^{+}(32 \mathrm{amu})$ between $90-$ $140 \mathrm{~km}$ (e.g., Shunk and Nagy, 2000) and heavy cluster ions (with amu such as $37,55,83, \ldots$ ) below $90 \mathrm{~km}$ - negative ions are only important below $60 \mathrm{~km}$ (Friedrich, 2004). Note that $\mathrm{O}^{+}$starts to appear above $130 \mathrm{~km}$. In addition, for the equatorial ionosphere below $140 \mathrm{~km}$, electron and ion temperatures are expected to be equal, i.e., $T_{e}=T_{i}$.

To fit our measured Doppler spectra from this region of the ionosphere we made use of the following spectral models:

1. Collisional incoherent scatter (IS) model: Although incoherent scatter spectral theory is well known - see, e.g., Farley (1971) for a comprehensive review - we outline here its pertinent aspects. The backscattered signal spectrum from an ionosphere in thermal equilibrium 
is known to be (e.g., Swartz and Farley, 1979) proportional to the real part of

$$
\frac{\left|\mu y_{i}+j k^{2} \lambda_{e}^{2}\right|^{2} \frac{y_{e}}{\omega-\mathbf{k} \cdot \mathbf{V}_{e}}+\left|y_{e}\right|^{2} \frac{y_{i}}{\omega-\mathbf{k} \cdot \mathbf{V}_{i}}}{\left|y_{e}+\mu y_{i}+j k^{2} \lambda_{e}^{2}\right|^{2}}
$$

where $k \equiv|\mathbf{k}|$ is the Bragg wavenumber (twice the wavenumber of backscattered radiowave), $\lambda_{e}$ the electron Debye length, $\mu \equiv T_{e} / T_{i}$, and, in case of multiple ion species an ion-composition weighted sum of $\mu y_{i}$ and $\frac{y_{i}}{\omega-\mathbf{k} \cdot \mathbf{V}_{i}}$ over all ion species is implied; $y_{e, i}$ stand for normalized admittances (e.g. Farley et al., 1961) for electrons and ions which are functions of wave frequency $\omega$ and Bragg vector $\mathbf{k}$ as well as the state parameters of ionospheric plasma. In the lower ionosphere, where collisions with neutrals are a dominant process, the use of the BGK collision model (Bhatnagar et al., 1954) leads to an admittance model (Dougherty and Farley, 1963)

$y_{s}=j+\theta_{s} \frac{J_{s}}{1-\psi_{s} J_{s}}$

where

$J_{s} \equiv \int_{0}^{\infty} d t e^{-j \theta_{s} t} e^{-\left[\psi_{s} t+\frac{t^{2}}{4} \sin ^{2} \alpha+\frac{1}{\phi_{s}^{2}} \sin ^{2}\left(\frac{\phi_{s} t}{2}\right) \cos ^{2} \alpha\right]}$

is referred to as Gordeyev integral; the parameters $\theta_{s}$, $\psi_{s}$, and $\phi_{s}$ are the Doppler shifted wave frequency $\omega-\mathbf{k} \cdot \mathbf{V}_{s}$, collision frequency $v_{s}$, and gyro-frequency $\Omega_{s}$, respectively, all of them normalized by $\sqrt{2} k C_{s}$, with $C_{s} \equiv \sqrt{K T_{s} / m_{s}}$, where $K$ is Boltzmann constant, denoting the thermal speed of species $s$ of a bulk velocity $\mathbf{V}_{s}$, mass $m_{s}$, and temperature $T_{s}$.

At low frequencies $\omega$ and within the altitude regime probed in this study the above model is primarily sensitive to ion parameters $T_{i}, \psi_{i} \propto v_{i}$, and $\mathbf{k} \cdot \mathbf{V}_{i}$; the gyrofrequencies and electron collisions cause no detectible variations in spectral shapes within the same regime. It is then convenient to consider a single ion case with $31 \mathrm{amu}$ mass (representing a mix of $\mathrm{NO}^{+}$and $\mathrm{O}_{2}^{+}$), and carry out a four parameter least-squares fit of the BGK IS model outlined above to the measured spectra; the fit parameters consist of the ion parameters mentioned above - Doppler shift $\mathbf{k} \cdot \mathbf{V}_{i}$, collision frequency $v_{i}$, and temperature $T_{i}$ - plus a scaling constant related to the background electron density and transmitted radar power (see Chau and Woodman, 2005). We looked into the possibility of fitting for ion composition, but given that the major ion components have similar masses the spectrum shape is almost invariant even to drastic changes of composition, at least for $z<100 \mathrm{~km}$.
2. Lorentzian model: For $k^{2} \lambda_{e}^{2} \ll 1$ and in the high collision frequency limit $\psi_{i} \gg 1$ applicable at lower altitudes, the IS spectral shape above approaches (e.g., Dougherty and Farley, 1963; Tanenbaum, 1968) a simple Lorentzian

$\frac{1}{1+\left(\omega-\mathbf{k} \cdot \mathbf{V}_{i}\right)^{2} \tau_{d}^{2}}$,

where

$\tau_{d} \equiv \frac{v_{i} m_{i}}{2 K T_{i} k^{2}}$

represents the ion diffusion time at the Bragg scale. Using this Lorentzian model for the measured spectra we fit for a scaling constant related to the received power as well as for Doppler shift $\mathbf{k} \cdot \mathbf{V}_{i}$ and the diffusion time $\tau_{d}$. A similar approach is also used with Arecibo ISR data from the lower ionosphere (e.g., Mathews, 1986, and references therein). Note that one can calculate $T_{i}$ from the measured $\tau_{d}$ if $v_{i}$ is known, and vice versa. For instance, in Sect. 5, we obtain $T_{i}$ from fitted $\tau_{d}$ using a collision frequency model based on Richmond (1972). The model equations for ion-neutral collisions are

$$
\begin{aligned}
v_{\mathrm{NO}^{+}}= & \frac{10^{-22} q}{m_{\mathrm{NO}^{+}}}\left\{1.07\left[\mathrm{~N}_{2}\right]\left(\frac{T}{T_{0}}\right)^{-0.16}+\right. \\
& \left.1.06\left[\mathrm{O}_{2}\right]\left(\frac{T}{T_{0}}\right)^{-0.16}+0.6[\mathrm{O}]\left(\frac{T}{T_{0}}\right)^{-0.19}\right\},(6) \\
v_{\mathrm{O}_{2}^{+}}= & \frac{10^{-22} q}{m_{\mathrm{O}_{2}^{+}}}\left\{0.89\left[\mathrm{~N}_{2}\right]\left(\frac{T}{T_{0}}\right)^{-0.20}+\right. \\
& \left.1.16\left[\mathrm{O}_{2}\right]\left(\frac{T}{T_{0}}\right)^{0.05}+0.89[\mathrm{O}]\left(\frac{T}{T_{0}}\right)^{0.36}\right\},(7)
\end{aligned}
$$

where $T_{0} \equiv 500 \mathrm{~K}, T$ is the neutral temperature taken from the MSISE-90 model (e.g., Hedin, 1991), [X] stands for the neutral density of atom or molecule X, and $q$ is the magnitude of electron charge, all in MKS units. Since we are fitting for an average ion component, we used an averaged ion-neutral collision frequency corresponding to the arithmetic mean of $v_{\mathrm{O}_{2}^{+}}$ and $v_{\mathrm{NO}^{+}}$. Note that the collision frequencies depend on neutral temperature $T$, and since $T=T_{i}$ in this altitude range, one could in principle directly invert the measured diffusion times for ion temperature $T_{i}$ using a suitable model for the neutral densities. However, that task will be left for future work.

A distortion is applied to the theoretical models described above prior to fitting them to the measured spectra in order to compensate for the windowing effect of finite-length timeseries used in spectral estimation. A weighted least-squares fitting is then carried out with the Levenberg-Marquardt algorithm (e.g., Bevington, 1969) using a procedure similar to those described in Kudeki et al. (1999) and Chau (1998). 
(a) Spectrogram with EEJ [dB]

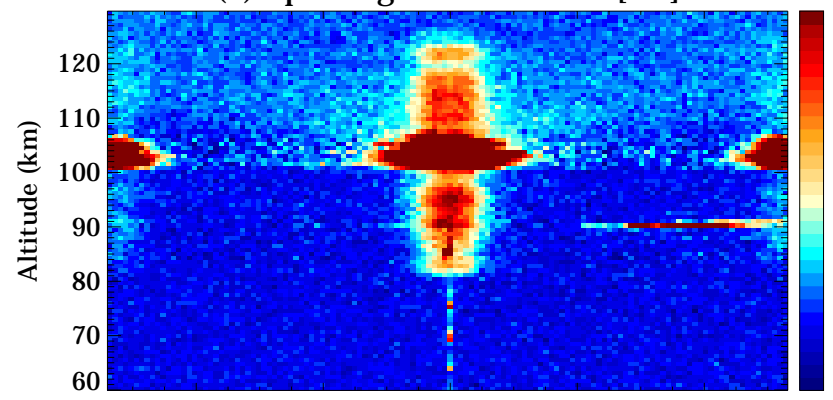

(b) Spectogram without EEJ [dB]

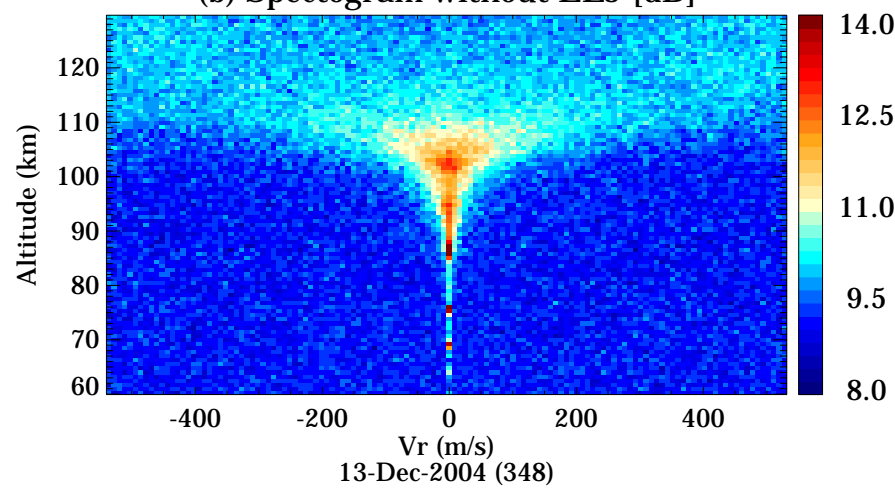

Fig. 1. Examples of spectrograms obtained under the presence (absence) of EEJ echoes at the top (bottom) obtained on 13 December 2004 at 17:27 (17:07) LT. Each spectrogram have been integrated 3 min. The color represents relative total power in $\mathrm{dB}$ (see color bar) between 60 and $130 \mathrm{~km}$.

\section{Radar observations}

The new results to be shown in this section were observed in the absence of strong EEJ echoes. However, to help the reader understand the effects of EEJ returns normally present in JRO radar experiments we include in Fig. 1a a Doppler spectrogram obtained during a period with strong EEJ echoes (top panel). The same figure also includes a second spectrogram (bottom panel) recorded when there were no EEJ echoes. In both spectrograms the color scale represents the relative total power (backscattered signal plus noise) in $\mathrm{dB}$ while the horizontal and vertical axes represent radial velocity (positive towards the radar) in $\mathrm{m} / \mathrm{s}$ and the scattering altitude in $\mathrm{km}$, respectively. The spectrograms are constructed from Doppler spectra (see Fig. 4 for examples of individual spectra) obtained with 3-min integration of 128-point periodograms. The periodograms are, in turn, the magnitude square of 128-point FFT of time-series samples collected on a pulse-to-pulse basis.

When EEJ echoes are present, as shown in Fig. 1a, they practically wipe out the weak ISR component of the returns, not only in the region they appear $(100-105 \mathrm{~km})$, but also at lower and higher altitudes due to range sidelobes of the coded transmitter pulses. It should be clarified here that (a) Measured Spectrogram [dB]

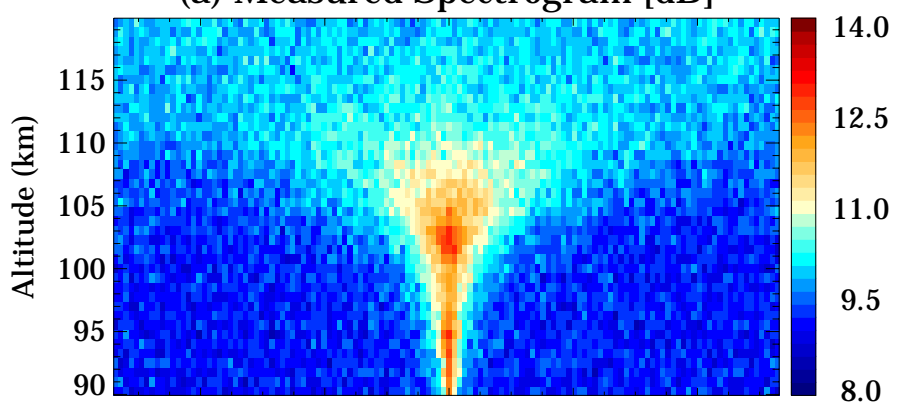

(b) 1-ion Fit Spectrogram [dB]

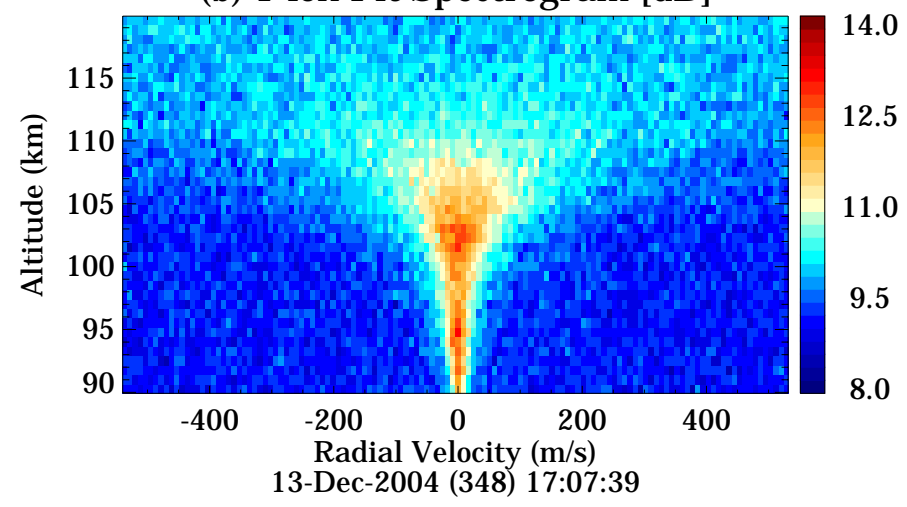

Fig. 2. Similar to Fig. 1, but this time the top panel shows the measured spectrogram, and the bottom panel shows the fitted spectrogram. Note that we have added the expected uncertainties to the fitted spectrogram.

the saturated signals seen in Fig. 1a at the left and right edges correspond to cross-talk echoes from the orthogonal circular polarization, which is phase-flipped on every other pulse transmission so as to avoid the interference of the opposing polarized returns within the baseband (i.e., near zero Doppler frequency) (e.g., Farley, 1969). Coming to our main results, Fig. 1b shows not only an example of the first Eand D-region ISR spectra recorded at Jicamarca, but also the cleanest E-region spectra of all the ISRs, given that Jicamarca's VHF frequency allows pulse-to-pulse estimation of the spectra. Note that such estimation is only good up to $\sim 110 \mathrm{~km}$, above this height the ISR spectra get wider causing frequency aliasing.

In Fig. 2 we compare the measured ISR spectrogram (top panel) for the 90 to $120 \mathrm{~km}$ altitude range with a synthetic spectrogram (bottom) obtained by fitting the theoretical BGK IS model to the measured spectra. In order to make a closer comparison, we have added a random noise component to the fitted spectrogram with an rms value that depends on the number of incoherent integrations and the fitted value. From a simple inspection, the agreement is excellent, moreover the goodness of fit is also excellent.

Given that the ISR spectrum gets narrower with decreasing altitude it is possible to use at lower altitudes coherent 
integration with the sampled data that has an effect of improving the overall signal-to-noise ratio (SNR) - the technique, much used in MST radar applications, is convenient when the sampling interval is much smaller than the correlation time of the signal component. In Fig. 3a we show a lowaltitude spectrogram example constructed using 32-point coherent integration. 128-point time-series of coherently integrated samples were Fourier transformed in peridogram construction, and 66 consecutive periodograms were averaged to obtain the spectra stacked in Fig. 3a; the overall integration time of the spectrogram shown in Fig. 3a is about $12 \mathrm{~min}$.

Figure $3 b$ shows a synthetic spectrogram constructed from Lorenztian model fits to the spectra of Fig. 3a. As mentioned in Sect. 3, when the ISR spectrum is dominated by collisions, it is well represented by the Lorentzian curve Eq. (4). Note that there is a good agreement between the measured and the fitted spectrograms, except for those regions were fitting was not possible (e.g., due to lack of convergence). The saturated red regions represent signals from coherent echoes (from meteors around $85 \mathrm{~km}$, and from mesospheric echoes below $80 \mathrm{~km}$ ). The weak ISR signals are seen as the light blue shades in the figure, which are of course the most exciting and novel aspect of this data set.

We have applied both the collisional ISR and Lorentzian fitting procedures to all the altitudes of interest between 60 and $120 \mathrm{~km}$ using different numbers of coherent integrations over overlapping altitude regions. More specifically, we used 8 (32) coherent integrations between 80 and $100 \mathrm{~km}(60$ $90 \mathrm{~km}$ ). Fitting a Lorentzian function is of course much faster than fitting a numerically evaluated ISR spectrum. In Fig. 4, we show examples of individual Doppler spectra (i.e., "cuts" from the spectrograms shown in Figs. 2 and 3) comparing the different fitting procedures. The measured spectra are shown in red, while the BGK IS and Lorentzian fits are in green and and blue, respectively, and virtually impossible to distinguish from one another at the scale shown, in particular at lower altitudes. Only above $106 \mathrm{~km}$ some very small discrepancies start to appear, but they are so small that both fits have about the same goodness of fit value.

\section{E- and D-region parameters inferred from the mea- sured spectra}

In this section we present the profiles of ionospheric parameters obtained by fitting the measured spectra with the spectral models described in Sect. 3. The results of the fits to the single-ion BGK IS model are shown in Fig. 5. Each panel of the figure exhibits one of the four fit parameters of the single-ion model, namely, the relative signal power, ion drift velocity, ion-neutral collision frequency $v_{i}$, and temperature $T_{i}$. In panels (c) and (d) the orange lines represent $v_{i}$ and $T$ from the MSISE-90 model. Note that the electron densities at these heights were estimated by Chau and Woodman (2005) by combining the relative power measurements from (a) Measured Spectrogram [dB]

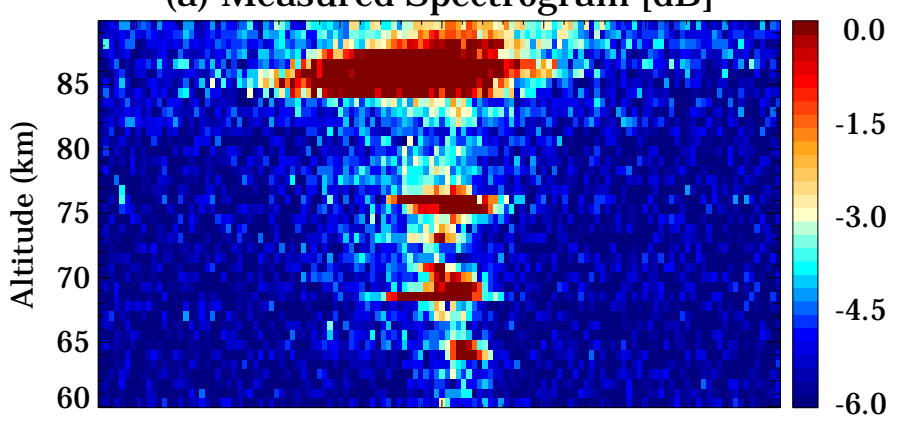

(b) Lorentzian Spectrogram [dB]

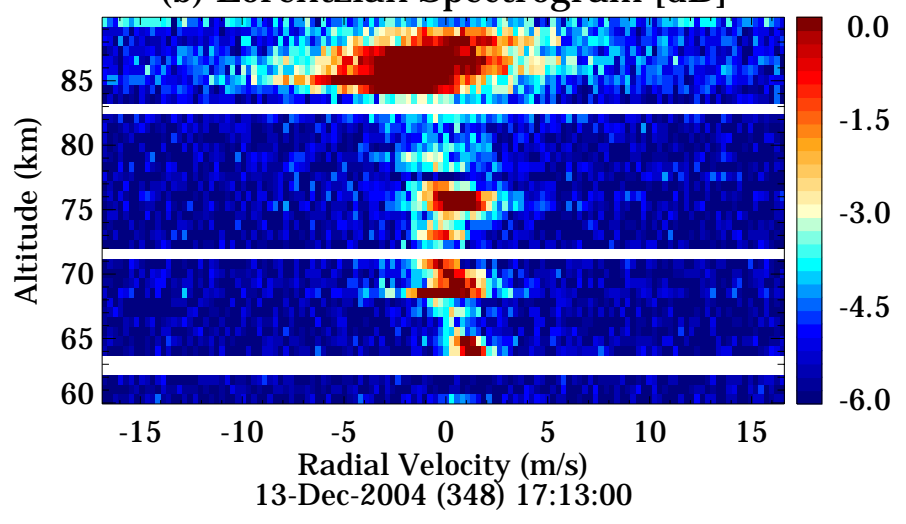

Fig. 3. D-region measured (fitted) spectrogram at the top (bottom) obtained on 13 December 2004 around 17:13 LT. Each spectrum was obtained with $\sim 12$ min averaging. The horizontal white lines in (b) mark the alitudes where fitting failed.

both circular polarizations and normalizing the sum against Faraday rotation densities at F-region peak.

Excluding the obvious outliers, the Doppler velocities shown in Fig. 5b exhibit relatively small random errors of about $\sim 1 \mathrm{~m} / \mathrm{s}$ magnitude below $100 \mathrm{~km}$ and somewhat larger values at higher altitudes where the spectra broaden. Above $110 \mathrm{~km}$ the estimated velocities are more variable due to frequency aliasing. Also, above $87 \mathrm{~km}$, both $v_{i}$ and $T_{i}$ estimates are in excellent agreement with MSISE-90. However, the results below $87 \mathrm{~km}$ show discrepancies with MSISE-90 which will be discussed later in this section.

In Fig. 6 we show the results of fitting the measured spectra to the Lorentzian model parametrized by (a) relative power, (b) Doppler velocity, and (c) diffusion time $\tau_{d}$ defined in Eq. (5). We also plot in panel (d) the ion temperature $T_{i}$ derived from diffusion time $\tau_{d}$. Recall that $T_{i}$ is obtained from $\tau_{d}$ using the collision frequencies $v_{i}$ taken from the Richmond and MSISE-90 models.

Panels (a) and (b) in Figs. 5 and 6 are very similar and consistent with one another and require no further elaboration. The derived temperature shown in Fig. 6d is in a reasonable agreement with the estimated temperature of Fig. 5d between 87 and $100 \mathrm{~km}$ altitudes. But, above $100 \mathrm{~km}$, the 

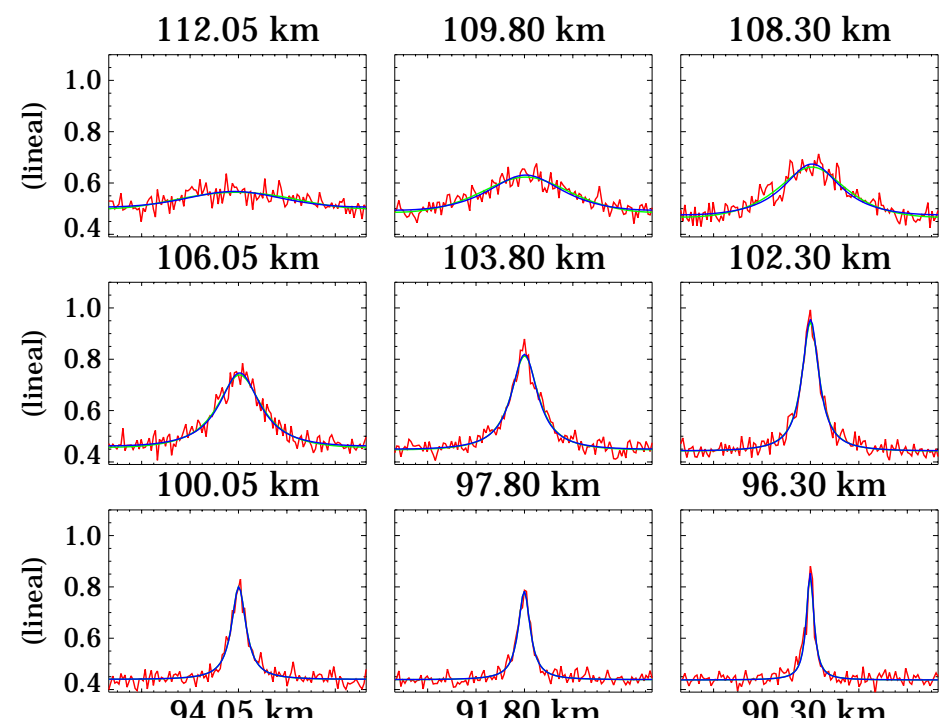

$96.30 \mathrm{~km}$
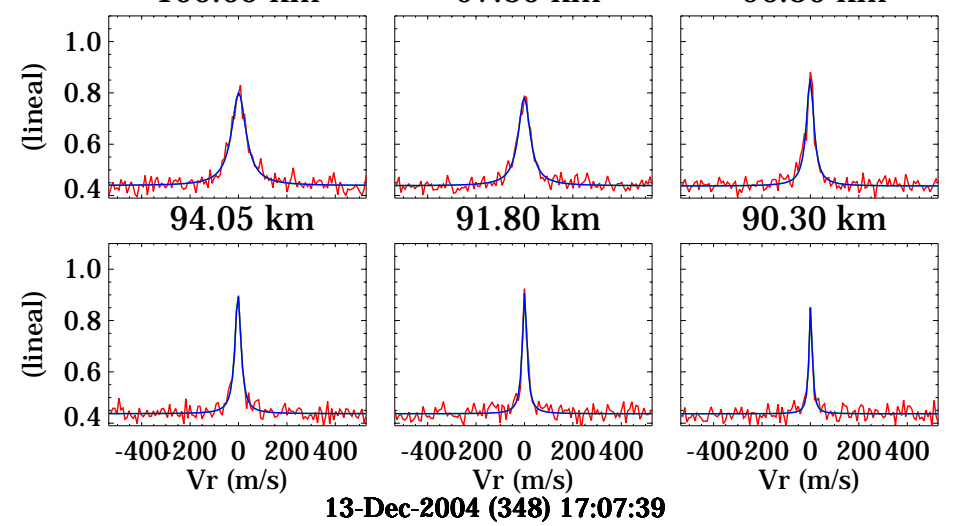

Fig. 4. Comparisons of Doppler spectra at E-region heights; measured spectra (red), single-ion ISR spectra (green), and Lorentzian spectra (blue).

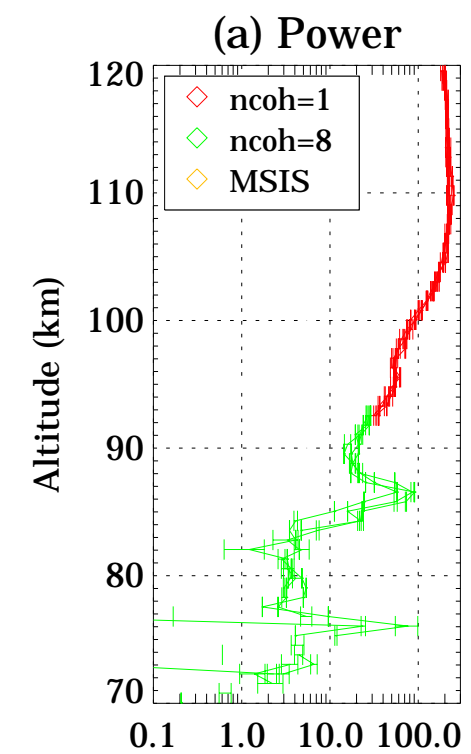

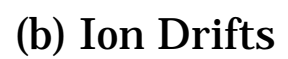

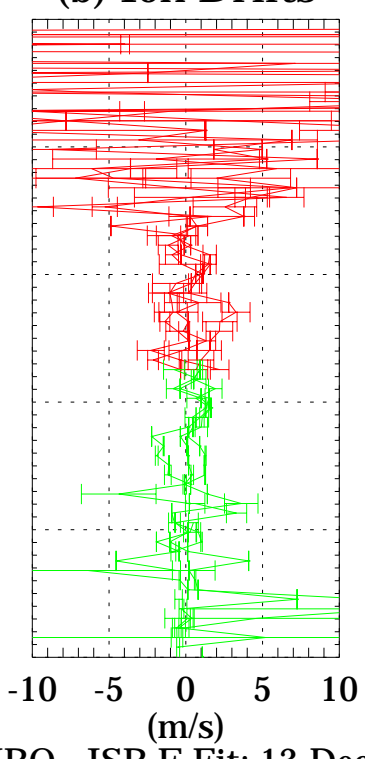

(c) I on collisions

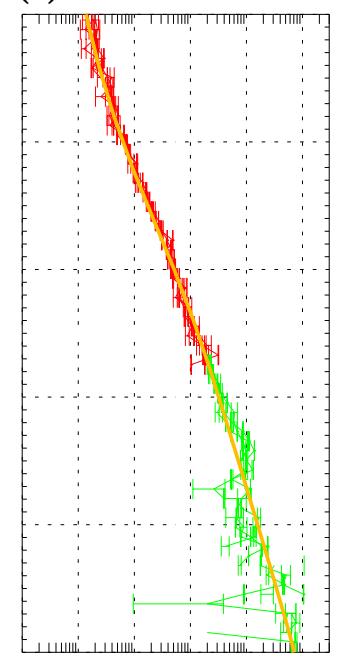

$10^{1} 10^{2} 10^{3} 10^{4} 10^{5} 10^{6}$

Frequency $(\mathrm{Hz})$ (d) $\mathrm{Ti}, \mathrm{Te}$

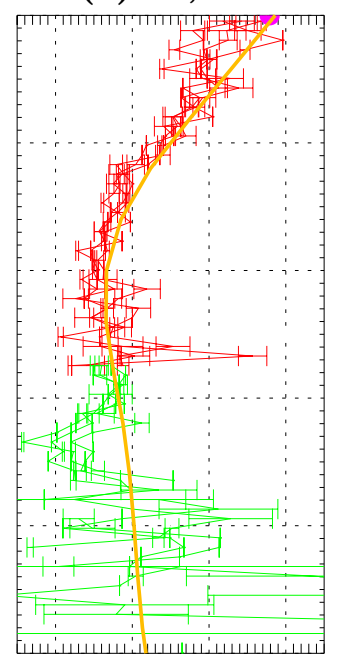

100200300400

( $\mathrm{K}$ )

Fig. 5. Profiles of ISR parameters obtained from fitting the single-ion ISR spectrum: (a) Relative power, (b) Doppler velocity, (c) ion-neutral collision frequency, and (d) ion/electron temperature. Profiles in red (green) were obtained with 1 (8) coherent integrations every $\sim 3$ min. The orange lines in (c) and (d) show MSISE-90 based collision frequencies and temperatures. 
(a) Power

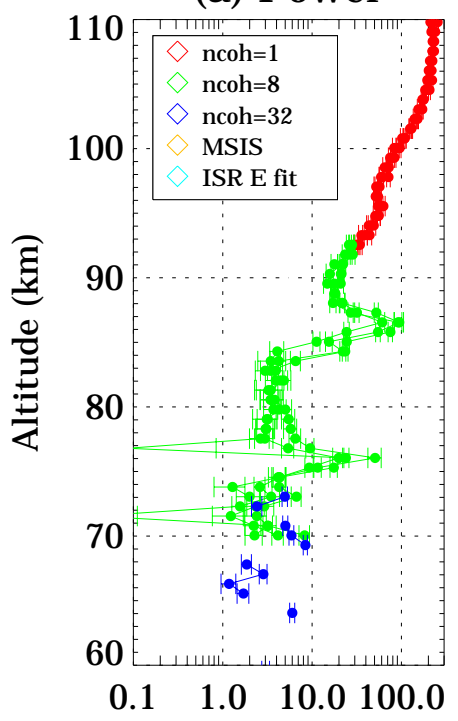

(b) I on Drifts

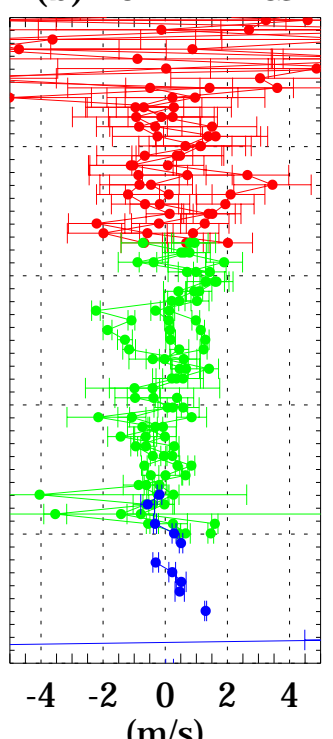

(c) Diffusion Time

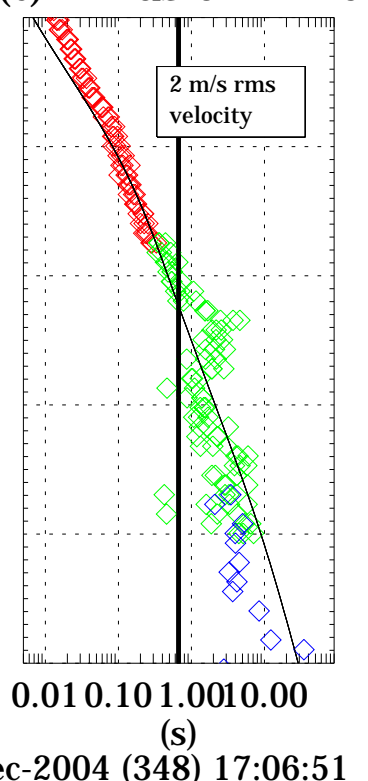

(d) $\mathrm{Ti}, \mathrm{Te}$

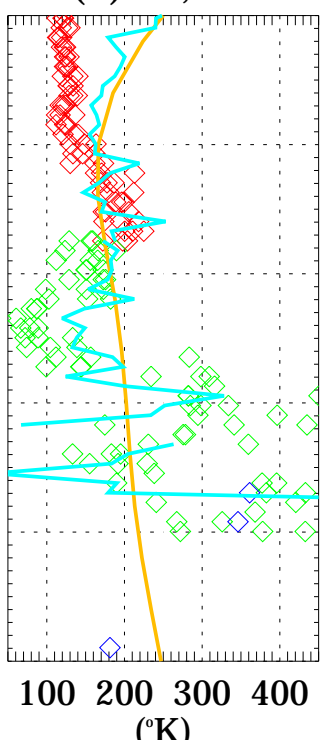

J RO - Lorentzian Fit: 13-Dec-2004 (348) 17:06:51

Fig. 6. Profiles of ISR parameters obtained from fitting a Lorentzian spectrum: (a) Relative power, (b) Doppler velocity, (c) diffusion time, and (d) ion/electron temperature. Profiles in red (green) were obtained with 1 (8) coherent integrations every $\sim 3$ min. Profiles in blue, were obtained with 32 coherent integrations every $\sim 12 \mathrm{~min}$. The black lines in (c) represent diffusion time of a $2 \mathrm{~m} / \mathrm{s}$ rms turbulence (vertical), and the expected diffusion time from MSISE-90 parameters. In (d) the orange line shows the MSISE-90 temperature and the light blue line the averaged temperature from fitting ISR spectrum (Fig. 5d).

derived temperature is lower than the MSISE-90 temperature shown in orange and the estimate from Fig. $5 \mathrm{~d}$ re-drawn in blue. The lower temperature estimates in Fig. 6d above $100 \mathrm{~km}$ are a clear indication that although the Lorentzian model fits the measured spectra very well the interpretation of diffusion time is not as simple as given by Eq. (5) at those higher altitudes. At these altitudes, collisions with neutrals start to become less dominant, and the full ISR spectrum model is needed to get reliable temperature estimates such as those shown in Fig. 5d.

All the parameter profiles in Figs. 5 and 6 except for the drift velocity profiles exhibit an extremum centered about $87 \mathrm{~km}$ altitude, such as the temperature minimum at about $100 \mathrm{~K}$ seen in Fig. 5d. These localized features are a consequence of a long duration meteor echo which contaminated the incoherent scatter results. Note that below $87 \mathrm{~km}$ the temperature estimates obtained by both fitting methods appear to be somewhat larger than the MSISE-90 temperatures. Of course below $87 \mathrm{~km}$ there are layers of coherent echo enhancements as indicated by enhanced power levels in Figs. 5a and $6 \mathrm{a}$ (see also the saturated red regions in Fig. 3). However, both coherent and incoherent (weak) spectra show comparable spectral widths (diffusion times) at these altitudes. Compared to the expected ion diffusion time (using MSISE-90 parameters, and shown as the thin black line in Fig. 6c), the measured diffusion times are generally smaller (i.e., wider spectral widths) here.

Similar results, i.e., wider spectral widths below $80 \mathrm{~km}$, have also been observed over Arecibo (e.g., Harper, 1978; Fukuyama, 1981). At Arecibo, these broadening effects were attributed to the presence of negative ions, that could broaden the spectra as much as $100 \%$ (e.g., Mathews, 1978). Nonetheless, recent advances in the understanding of chemistry and physics of D-region indicate that negative ions should only be important below $60 \mathrm{~km}$ in the daytime (e.g., Kazil et al., 2003). At Jicamarca, spectral widths in the Dregion have been measured for many years from coherent scatter echoes. The widths have been mainly attributed to the combined effects of atmospheric turbulence, gravity waves, and in cases when wide beams were used, to an effect known as beam broadening. Based on these previous studies, we suggest that the measured small diffusion times (wider spectra) of the D-region ISR returns presented here are probably due to the same spectral broadening effects affecting the coherent echoes (e.g., Woodman and Guillén, 1974), particularly atmospheric turbulence. 


\section{Conclusions}

We have shown in this paper that when coherent echoes from EEJ irregularities are not present, very accurate ISR spectral measurements can be made at Jicamarca using a pulseto-pulse estimation technique. Two types of spectral fitting functions were examined: a single-ion ISR spectrum model based on BGK collisions and a Lorentzian spectrum parametrized by a diffusion time. As expected, in both cases very precise Doppler shift estimates (e.g., less than $1 \mathrm{~m} / \mathrm{s}$ uncertainty below $100 \mathrm{~km}$ ) were obtained. Above $87 \mathrm{~km}$ spectral fitting of the ISR model provides reasonable values for ion-neutral collisions and ion temperatures. Between 87 and $100 \mathrm{~km}$, Lorentzian fitting also provides reliable temperatures inferred from diffusion times and collision frequency models, but higher up, above $100 \mathrm{~km}$, the interpretation of the measured spectral widths in terms of a simple ion-diffusion time seems to be problematic. Also, below $87 \mathrm{~km}$, i.e., in the D-region, the measured diffusion times are smaller than expected. Both spectral models lead to temperature estimates larger than those predicted by MSISE-90 . We suggest that this effect is due to a Doppler spectral broadening caused by relatively large turbulent motions known to exist at these altitudes.

We plan to conduct further measurements of the type reported here during counter-electrojet periods to be encountered in the near future. Based on the available statistics of zonal electric fields counter-electrojet events are known to be frequent in December solstice months in the afternoon hours (e.g., Fejer et al., 1991). New and longer period measurements that we hope to conduct, should help validate and extend the D-region results reported in this paper.

Acknowledgements. The authors thank D. Farley, D. Hysell, J. Mathews, M. Milla, M. Sulzer, R. Woodman, and Q. Zhou for their comments and suggestions during early stages of this work. The MSISE-90 results were obtained from the web pages of NASA's National Space Science Data Center. The Jicamarca Radio Observatory is a facility of the Instituto Geofísico del Perú and is operated with support from the NSF Cooperative Agreement ATM0432565 through Cornell University. This research was funded at the University of Illinois by the NSF grant ATM-0215246.

Topical Editor M. Pinnock thanks D. Farley and G. Lehmacher for their help in evaluating this paper.

\section{References}

Bevington, P. R.: Data Reduction and Error Analysis for the Physical Sciences, McGraw Hill, 1969.

Bhatnagar, P. L., Gross, E. P., and Krook, M.: Model for Collision Processes in: Gases I, Small Amplitude Processes in Charged and Neutral One-Component Systems, Phys. Rev., 94, 511-525, 1954.

Bowles, K. L., Ochs, G. R., and Green, J. L.: On the Absolute Intensity of Incoherent Scatter Echoes from the Ionosphere, J. of Res. NBS - D. Rad. Prop., 66D, 395-407, 1962.
Chapin, E. and Kudeki, E.: Radar Interferometric Imaging Studies of Long-Duration Meteor Echoes Observed at Jicamarca, J. Geophys. Res., 99, 8937-8949, 1994.

Chau, J. L.: Examination of various techniques for measuring wind velocities using clear-air radars, with emphasis on vertical wind measurements, Ph.D. thesis, Univ. of Col., Boulder, 1998.

Chau, J. L.: Unexpected Spectral Characteristics of VHF Radar Signals from 150-km Region over Jicamarca, Geophys. Res. Lett., 31, L23 803, doi:10.1029/2004GL021620, 2004.

Chau, J. L. and Woodman, R. F.: Observations of Meteor-Head Echoes Using the Jicamarca $50 \mathrm{MHz}$ Radar in Interferometer Mode, Atmos. Chem. Phys., 4, 511-521, 2004.

Chau, J. L. and Woodman, R. F.: D and E region incoherent scatter radar density measurements over Jicamarca, J. Geophys. Res., 110, A12 314, doi:10.1029/2005JA011438, 2005.

Dougherty, J. P. and Farley, D. T.: A Theory of Incoherent Scattering of Radio Waves by a Plasma, J. Geophys. Res., 68, 54735486, 1963.

Farley, D. T.: Faraday Rotation Measurements Using Incoherent Scatter, Radio Sci., 4, 143-152, 1969.

Farley, D. T.: Radio wave scattering from the ionosphere, Methods of Experimental Physics, 9, 139-186, 1971.

Farley, D. T.: Theory of Equatorial Electrojet Plasma Waves: New Developments and Current Status, J. Atmos. Sol. Terr. Phys., 47, 729-744, 1985.

Farley, D. T.: Early Incoherent Scatter Observations at Jicamarca, J. Atmos. Sol. Terr. Phys., 53, 665-675, 1991.

Farley, D. T., Dougherty, J. P., and Barron, D. W.: A Theory of Incoherent Scattering of Radio Waves by a Plasma II. Scattering in a magnetic field, Proc. Phys. Soc. London, 263, 238-258, 1961.

Fejer, B. G., de Paula, E. R., Gonzalez, S. A., and Woodman, R. F.: Average Vertical and Zonal $F$ Region Plasma Drifts over Jicamarca, J. Geophys. Res., 96, 13 901-13 906, 1991.

Friedrich, M.: The Lower Ionosphere: Abandoned by Communications, to Be Re-Discovered for aeronomy, The Rad. Sci. Bulletin, 309, 38-45, 2004.

Fukuyama, K.: Incoherent Scatter Radar Observations of WaveLike Structures in the Mesosphere over Arecibo, J. Geophys. Res., 86, 9152-9158, 1981.

Gray, R. W. and Farley, D. T.: Theory of Incoherent-Scatter Measurements Using Compressed Pulses, Radio Sci., 8, 123-131, 1973.

Harper, R. M.: Preliminary Measurements of the Ion Component of the Incohenrent Scatter Spectrum in the $60-90 \mathrm{~km}$ Region over Arecibo, Geophys. Res. Lett., 5, 784-786, 1978.

Hedin, A. E.: Extension of the MSIS Thermospheric Model Into the Middle and Lower Atmosphere, J. Geophys. Res., 96, 1159$1172,1991$.

Hysell, D. L.: Incoherent Scatter Experiments at Jicamarca Using Alternating Codes, Radio Sci., 35, 1425-1435, 2000.

Kazil, J., Kopp, E., Chabrillat, S., and Bishop, J.: The University of Bern Atmospheric Ion Model: Time Dependent Ions Modeling in the Mesosphere and Lower Thermosphere, J. Geophys. Res., 108, doi:10.1029/2002JDD003024, 2003.

Kudeki, E.: Radar interferometer observations of Mesospheric echoing layers at Jicamarca, J. Geophys. Res., 93, 5413-5421, 1988.

Kudeki, E. and Fawcett, C. D.: High resolution observations of $150 \mathrm{~km}$ echoes at Jicamarca, Geophys. Res. Lett., 20, 1987- 
1990, 1993.

Kudeki, E., Bhattacharyya, S., and Woodman, R. F.: A New Approach in Incoherent Scatter $F$ Region E x B Drift Measurements at Jicamarca, J. Geophys. Res., 104, 28 145-28 162, 1999.

Mathews, J. D.: The Effects of Negative Ions on ColiisionDominated Thomson Scattering, J. Geophys. Res., 83, 505-512, 1978.

Mathews, J. D.: Incoherent Scatter Radar Probing of the 60-100$\mathrm{km}$ Atmosphere and Ionosphere, IEEE Trans. Geosci. Remote Sensing, 24, 765-776, 1986.

Richmond, A. D.: Numerical Model of the Equatorial Electrojet, Tech. Rep. AFCRL-72-0668, ERP 421, Air Force Cambridge Res. Lab. Hanscom AFB, Bedford, Mass., 1972.

Sheth, R., Kudeki, E., Lehmacher, G., Sarango, M., Woodman, R. F., Chau, J. L., Guo, L., and Reyes, P.: A high-resolution study of mesospheric fine structure with the Jicamarca MST radar, Ann. Geophys., 24, 1281-1293, 2006.
Shunk, R. and Nagy, A. F.: Ionospheres: Physics, Plasma Physics, and Chemistry, Cambridge Univ. Press, New York, 570, 2000.

Swartz, W. E. and Farley, D. T.: A Theory of Incoherent Scattering of Radio Waves by a Plasma 5. The use of the Nyquist Theorem in general quasi-equilibrium situations, J. Geophys. Res., 84, 1930-1932, 1979.

Tanenbaum, B. S.: Continuum Theory of Thomson Scattering, Phys. Rev., 171, 215-221, 1968.

Woodman, R. F. and Chau, J. L.: Equatorial Quasiperiodic Echoes from Equatorial Field-Aligned Irregularities Observed over Jicamarca, Geophys. Res. Lett., 28, 207-209, 2001.

Woodman, R. F. and Guillén, A.: Radar observations of winds and turbulence in the stratosphere and mesosphere, J. Atmos. Sci., 31, 493-505, 1974. 